\title{
La Perspectiva de Género, Desafíos para la Ergonomía en Chile: Una Revisión Sistemática de Literatura
}

\section{THE GENDER PERSPECTIVE, CHALLENGES FOR ERGONOMICS IN CHILE: A SYSTEMATIC LITERATURE REVIEW}

\section{Pamela Astudillo Cornejo' ${ }^{1}$, Carlos Ibarra Villanueva²}

1. Ergónoma, Sección de Ergonomía, Subdepartamento de Salud de los Trabajadores, Departamento de Salud Ocupacional del Instituto de Salud Pública de Chile.

2. Ergónomo Asesor, Departamento de Salud Ocupacional, División de Políticas Públicas Saludables y Promoción, Subsecretaria de Salud Pública, Ministerio de Salud.

\begin{abstract}
RESUMEN
Contexto: Aun cuando la división sexual del trabajo remunerado ha sido examinada en muchos estudios, a veces considerada como la extensión de los roles diferenciados en el ámbito doméstico, el resultado de la jerarquía vertical donde los hombres se encuentran en los puestos superiores, la asociación de los hombres a las máquinas (distintas a las de limpieza o la costura) o la división de otras condiciones de trabajo y empleo por género. El género y el sexo rara vez son considerados en la investigación en ergonomía. Metodología: A través de una revisión de los datos publicados en encuestas chilenas con representación nacional, en bases de datos gubernamentales y artículos de ergónomos chilenos publicados en Ergonomics, Ciencia y Trabajo, etc. Objetivo: Este artículo busca orientar sobre las principales diferencias de sexo y género en los hombres y las mujeres para comprender sus implicancias en actividad de trabajo en Chile y cuál ha sido la contribución de la ergonomía a este aspecto. Conclusiones: El tomar en cuenta el sexo de las trabajadoras/es enriquece el análisis del trabajo y el análisis ergonómico a su vez enriquece la comprensión sobre la división sexual del trabajo y cómo esta genera diferentes exposiciones a los riesgos profesionales para las mujeres y los hombres.
\end{abstract}

(Astudillo P, Ibarra C, 2014. La Perspectiva de Género, Desafíos para la Ergonomía en Chile: Una Revisión Sistemática de Literatura. Cienc Trab. Ene-Abr; 16 [49]: 28-37).

Palabras clave: GÉNERO, SEXO, TRABAJO, ERGONOMÍA, CHILE, REVISIÓN DE LITERATURA

\section{INTRODUCCIÓN}

Este trabajo fue realizado en el marco del proyecto de cooperación internacional "Ergonomics For Health And Sustainable Development In Chile”, financiado por la Agencia Canadiense para el Desarrollo Internacional (ACDI), gestado por la Universidad de Quebec en Montreal (UQÀM) y el Instituto de Salud Pública de

\section{Correspondencia / Correspondence:}

Pamela Astudillo Cornejo

Sección Ergonomia, Subdepartamento Salud de los Trabajadores Departamento Salud Ocupacional - Instituto de Salud Pública de Chile Marathon 1000, Ñuñoa, Santiago, Chile

código postal 7780050

Tel.: +56 (2) 57289620

e-mail: pastudillo@ispch.cl

Recibido: 08 de Enero de 2014 / Aceptado: 06 Marzo 2014

\section{ABSTRACT}

Context: Even though the sexual division of paid work has been examined in many studies, sometimes thought of as the extension of differentiated roles in the domestic sphere, the result of a vertical hierarchy where men are found in higher positions, the association of men with machines (other than those used in cleaning or sewing), or a division of other working and employment conditions by gender. Gender and sex have rarely been considered in ergonomics research. Methodology: Through a review of published data from Chilean surveys with national representation, from governmental databases and published articles in Ergonomics, Ciencia y Trabajo, etc. Objective: This article aims to guide about the principals differences of sex and gender in men and women to understand the implications of sex and gender for work activity in Chile and what has been the contribution of ergonomics to this issue. Conclusions: Taking into account the sex of women and men workers enriches the analysis of work and the ergonomic analysis in turn enriches the understanding of the sexual division of work and how this generates different exposures to occupational risks for women and men.

Key words: GENDER, SEX, WORK, ERGONOMICS, CHILE, LITERATURE REVIEW

Chile. Corresponde a una revisión bibliográfica de artículos y encuestas nacionales con el propósito de describir las diferencias de sexo y de género en los trabajadores/as en Chile y cuál ha sido el aporte de la ergonomía en Chile al conocimiento de estas diferencias. Teniendo en cuenta que el análisis ergonómico es probablemente una de las herramientas más pujantes utilizada para intervenir en salud y seguridad del trabajo en países de América del Norte y Europa y considerando que la ergonomía es una disciplina que en Chile ya cuenta con más de 30 años de desarrollo, existiendo formación en nivel de pre- y posgrado en universidades. ${ }^{1,2}$

Aun cuando la división sexual del trabajo remunerado ha sido examinada en muchos estudios, a veces considerada como la extensión de los roles diferenciados al ámbito doméstico, el resultado de la jerarquía vertical donde los hombres se encuentran en los puestos superiores, la asociación de los hombres a las máquinas (distintas a las utilizadas en la limpieza o la costura) o la división de otras condiciones de trabajo y empleo por sexo. A pesar de las implicancias de esta división de la actividad laboral y de las inte- 
racciones humanas en el lugar de trabajo, el género y el sexo rara vez son considerados en la investigación en ergonomía en Chile y en otras partes del mundo.

Cabe mencionar que, conceptualmente, el "sexo" se refiere a la especificidad en las características biológicas de las mujeres y los hombres y el "género", se refiere a los factores sociales asociados con ser hombre o mujer. Sin embargo, en la investigación empírica con las poblaciones de los lugares de trabajo, a menudo resulta difícil distinguir si las diferencias observadas entre hombres y mujeres se deben a factores biológicos o sociales y, más aun, hasta qué punto los factores sociales logran modelar los factores biológicos. $^{3}$

En Chile, así como en el resto del mundo, la estructura del empleo ha sido marcada por la división sexual del trabajo, en sentido vertical y horizontal en la mayor parte de la industria, notablemente a causa de la influencia del taylorismo, lo que ha generado patrones de exposición a distintos factores de riesgo en hombres y mujeres de una manera diferenciada. Es por eso que la OIT ha considerado "La equidad de género" dentro de sus principios de trabajo decente para intentar, de cierta manera, corregir la inequidad de género que responde más bien a una construcción social que a factores netamente biológicos. Este trabajo busca entregar pistas de una situación existente en Chile y en el mundo, sobre la cual la ergonomía ya ha situado su interés, prueba de ello es el Comité Técnico de Trabajo y Género de la «International Ergonomics Association" (IEA), el número especial que la revista Ergonomics ha dedicado a este tema el año $2012^{4}$ o más recientemente la revista Laboreal. ${ }^{5}$

El presente artículo tiene como objetivo describir las diferencias de sexo y de género en los trabajadores/as chilenos y cuál ha sido el aporte de la ergonomía en la comprensión de estas diferencias a través de una revisión y evaluación de la productividad de investigación en la ergonomía chilena y la verificación de la incorporación de la perspectiva de género en las publicaciones.

\section{METODOLOGÍA}

El presente artículo corresponde a una revisión sistemática de literatura que utilizó diferentes fuentes bibliográficas, pero que se centra principalmente en dos búsquedas:

Búsqueda de identificación de encuestas y bases de datos gubernamentales: Fue realizada una revisión de las encuestas chilenas más importantes en materia de salud en el trabajo en el último tiempo (ENCLA 2011, ENCAVI 2006, ENETS 2009-2010) ${ }^{6-8}$, así como las bases de datos de la Superintendencia de Seguridad Social (SUSESO), los Ministerios de Salud (MINSAL) y del Trabajo (MINTRAB), el Instituto Nacional de Estadísticas (INE) y la Superintendencia de Pensiones, buscando identificar las diferencias de sexo y de género en la salud laboral y condiciones de trabajo de los trabajadores/as chilenos/as.

Búsqueda de publicaciones de autores chilenos: fue realizada una revisión sistemática de las publicaciones de ergónomos chilenos en revistas como Ergonomics, Applied Ergonomics, Works, Ciencia y Trabajo entre otras, a través de la búsqueda en bases de datos de Google scholar, Ebsco, Taylor \& Francis, SciELO, Medline, PubMed y Sciencedirect, para identificar si las publicaciones que toman en cuenta los aspectos de género en sus estudios o intervenciones y si estos son relevantes para comprender mejor las diferencias de sexo y de género de los trabajadores/as chilenos/as. Las palabras claves utilizadas en la búsqueda fueron: Ergonomía, género, sexo, trabajo, Chile / Ergonomics, gender, sex, work, Chile.

Criterios de inclusión: Artículos de estudios o intervenciones ergonómicas publicadas en revistas de ergonomía y/o salud del trabajo que cuenten con comité de revisión, que describan claramente el sexo de la población estudiada y el sector de actividad económica.

Criterios de exclusión: Estudios epidemiológicos, revisiones bibliográficas, publicaciones en revistas sin comité de revisión que no describan claramente el sexo de la población estudiada y el sector de actividad económica.

La búsqueda fue realizada durante el año 2013 y se centró en las publicaciones realizadas en el período enero 2000 a diciembre 2012. La revisión de cada artículo fue realizada por ambos autores; el procedimiento es detallado en la Figura 1.

Figura 1.

Diagrama del procedimiento seguido para selección de las publicaciones.

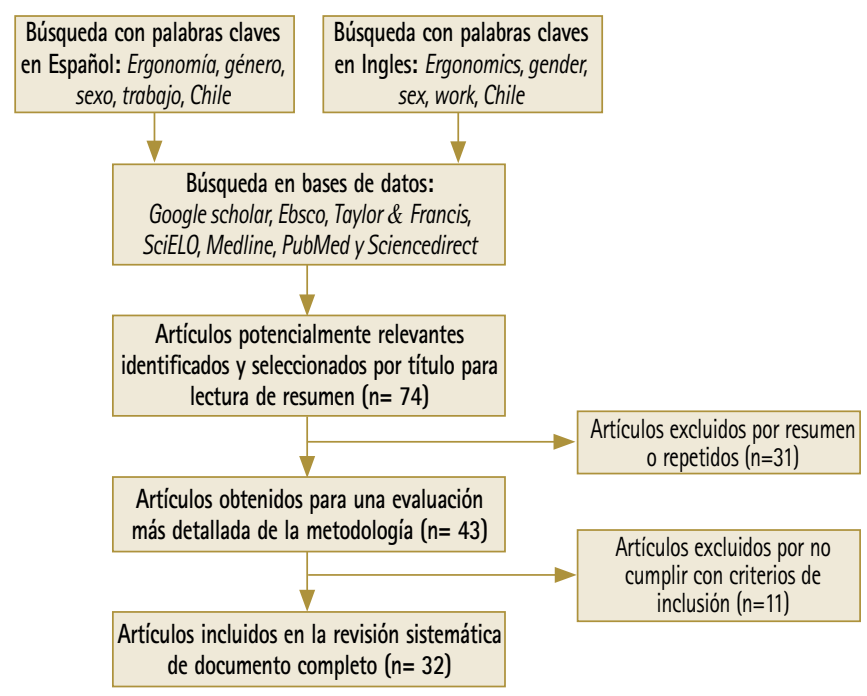

\section{RESULTADOS}

\section{Resultados revisión de encuestas y bases de datos gubernamentales}

Los datos presentados en la última encuesta de condiciones laborales del MINTRAB-INE ${ }^{9}$ muestran que existe un porcentaje de participación femenina en el empleo formal de un 40,3\% comparado con la participación masculina, que es del orden del 59,7\%, situación que se mantiene aun cuando el nivel de instrucción aumenta. Por otra parte, en la Figura 2 podemos observar que existe una importante brecha en el nivel de ingresos, en todas las categorías utilizadas en la ENETS 2009-2010 ${ }^{8}$, siendo mayor el porcentaje de mujeres en el tramo de ingresos más bajos (menos de \$133.600), contrariamente a lo que ocurre en el tramo de ingresos más elevado (más de \$851.000), donde los hombres son mayoría.

Cuando observamos la inserción laboral por tipo de actividad según los datos de la ENCLA $2011^{6}$, se puede constatar la segmentación de las ocupaciones según el género. La mayoría de las mujeres en Chile trabajan en puestos de trabajo que son socialmente considerados como femeninos: en las áreas de servicio 
Figura 2.

Nivel de ingresos según sexo. Modificado de ENETS 2009-2010.

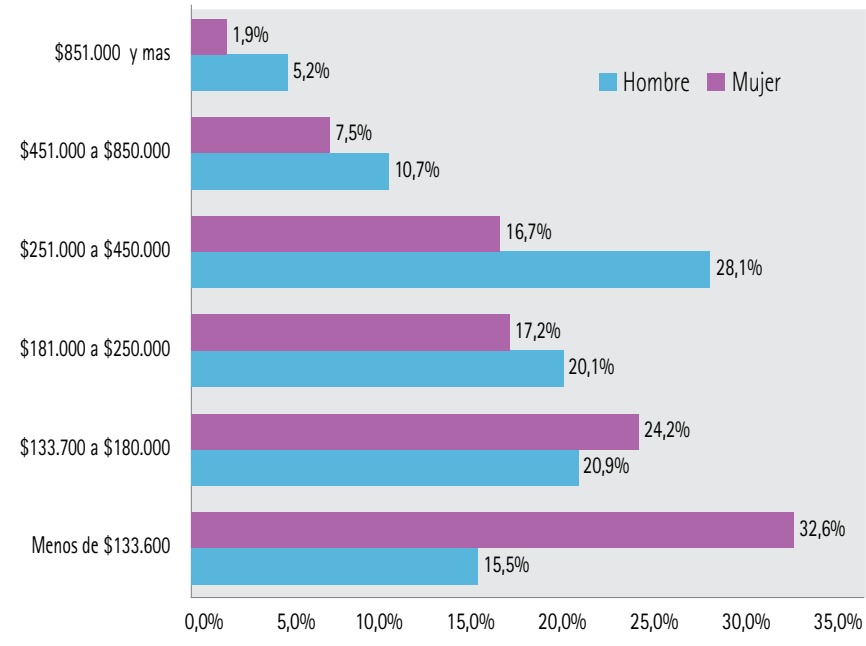

Figura 3.

Tasa de mortalidad laboral por accidentes del trabajo.

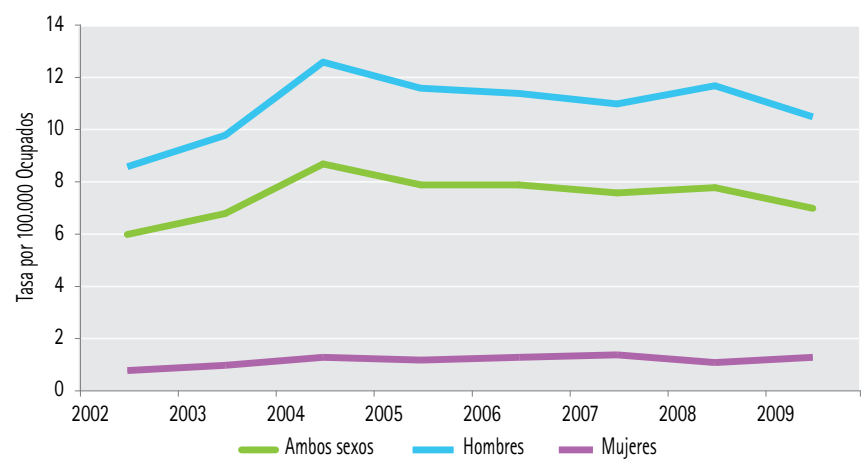

Fuente: DEIS MINSAL.

(64,4\%), enseñanza (60\%), intermediación financiera $(52,1 \%)$, hotelería y restaurantes $(49,6 \%)$ y comercio $(40,6 \%)$. Al contrario, los empleos masculinos presentan una distribución más heterogénea en todas las ramas de actividad económica, destacando la minería y la construcción, donde su participación supera el 90\%. Con respecto a la morbilidad y mortalidad de origen laboral, no existen antecedentes según sexo en las bases de datos públicas de la SUSESO y del MINSAL. Solo el MINSAL presenta datos de este tipo referentes a las tasas de accidentes del trabajo fatales desde al año 2002 al 2008, siendo la tasa de hombres muy superior a la de mujeres (Figura 3).

En cuanto a la percepción de la exposición a factores de riego (Figura 4), según datos de la ENETS 2009-2010, el 97,8\% de los hombres refieren exposición a factores de riesgo "ergonómicos», mientras que las mujeres, un 95,1\%. En ese contexto, el 67,7\% de las mujeres y el 73,3\% de los hombres refieren como un factor de riesgo importante el trabajo de pie, así como el 38,9\% de las mujeres y el 50,7\% de los hombres refieren tener que mantener la vista fija y concentrada.

Otro factor de riesgo mencionado ha sido el de trabajo repetitivo en cortos periodos de tiempo con un $27,7 \%$ para las mujeres y un $33,5 \%$ para los hombres. El trabajo sentado es percibido como un
Figura 4.

Exposición según tipo de riesgo ergonómico.

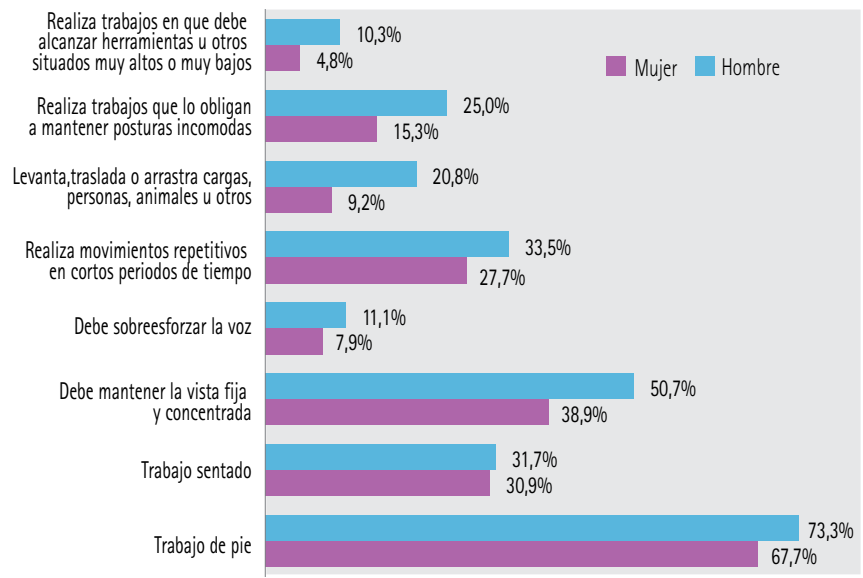

Fuente: ENETS 2009-2010.

Figura 5.

Lugar de los dolores provocados por el trabajo según sexo.

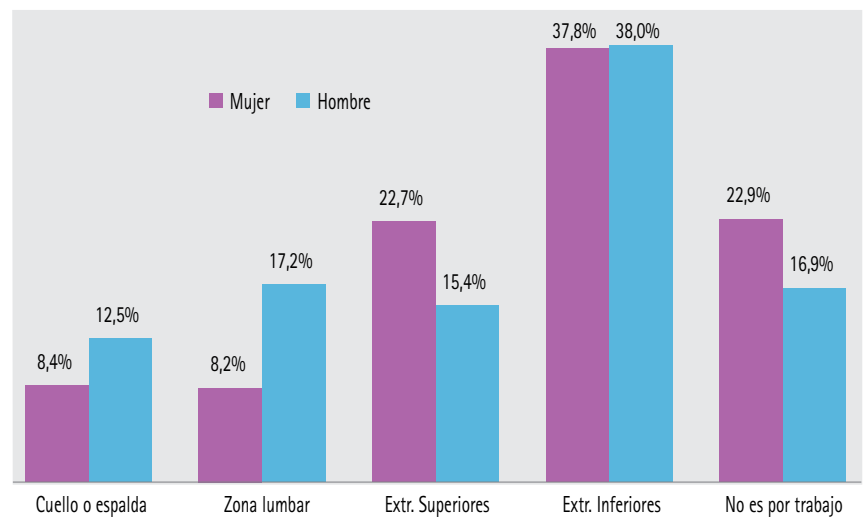

Fuente: ENETS 2009-2010.

factor de riesgo para un 30,9\% de las mujeres y un 31,7\% de los hombres. Con respecto a la manipulación manual de cargas, esta es referida como un factor de riesgo para el 20,8\% de los hombres y el 9,2\% de las mujeres encuestados/as.

Con respecto a la percepción de dolor en la Figura 5, podemos observar que en la ENETS 2009-2010 ${ }^{8}$ los trabajadores/as refieren principalmente dolor en las extremidades inferiores con un 38\% para los hombres y un 37,8\% para las mujeres. Cabe destacar, además, que las mujeres refieren un $22,7 \%$ de dolor en las extremidades superiores con respecto a un 15,4\% en los hombres y que la percepción de dolor en la zona lumbar es más elevada en los hombres, con un 17,2\% frente a un 8,2\% en las mujeres.

Respecto a la conciliación trabajo y familia, la ENCAVI $2006^{7}$ muestra que el 43,5\% de las mujeres considera que es la principal responsable del trabajo doméstico, mientras que el 55,1\% de los hombres refiere que solo realiza tareas puntuales. Lo que también es observado en la ENETS 2009-2010 ${ }^{8}$, donde las mujeres refieren realizar la mayor parte del trabajo doméstico, tendencia que es acentuada cuando la mujer se encuentra en el tramo de ingresos más bajo.

Por otra parte, según los datos publicados por la Superintendencia de Pensiones, en lo referente a la calificación de trabajos pesados desde 
el año 1998 al año 2009, se constató que la calificación de trabajos pesados se ha concentrado en sectores masculinizados como la minería (61\%), el transporte, almacenamiento y comunicaciones $(18 \%)$. Mientras que donde puede haber una mayor participación femenina el porcentaje de calificación es inferior: industria manufacturera (11\%), los servicios (9\%) y otros (1\%). A lo anterior se agrega que la distribución de los cotizantes beneficiados por calificación de trabajo pesado según sexo -en el período desde el año 1998 al año 2009-, el mayor porcentaje de beneficiarios a nivel nacional corresponde a hombres $(78,1 \%)$ en contraste con las mujeres $(21,9 \%)$, brecha que aumenta en la medida que la calificación corresponde a las regiones del norte del país donde la actividad minera es más importante, siendo para los cotizantes hombres del orden del 92,8\% en la II Región y de 92,9\% en la III Región.

\section{Resultados de la revisión sistemática de literatura}

Respecto de los hallazgos de la revisión, se puede constatar que la producción de publicaciones de ergónomos chilenos ha ido aumentando sostenidamente; no obstante, cabe señalar que las 32 publicaciones seleccionadas para la revisión correspondieron principalmente a estudios o intervenciones realizadas en trabajos donde predomina el sexo masculino, siendo 15 los estudios en población masculina, 8 en población femenina y 9 los que incluyen ambos sexos (Figura 6).

Figura 6.

Número de publicaciones según tipo de población bajo estudio.

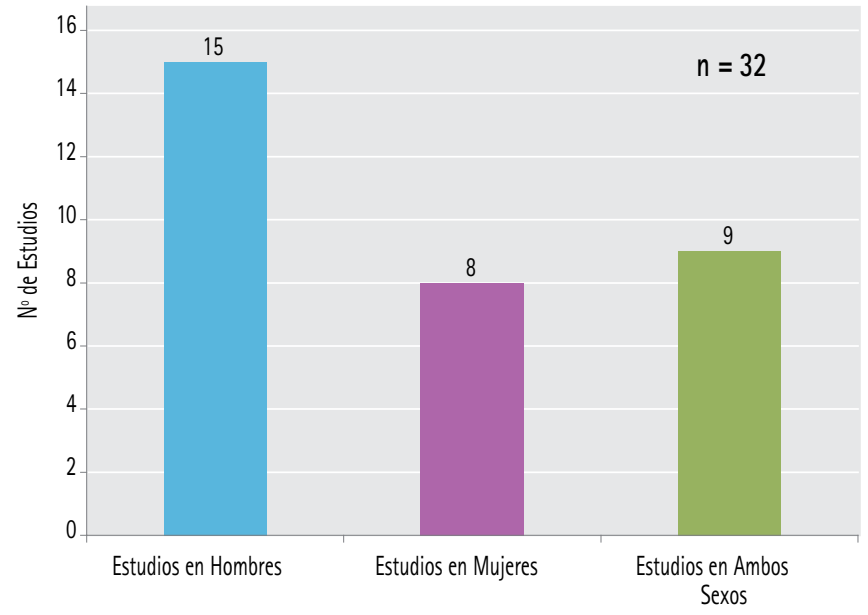

Cuando se analizó en detalle el contenido de las publicaciones, se tuvo en cuenta si los autores realizaban un análisis según sexo, vale decir, lo inherente a las características biológicas de los sujetos en estudio y luego si existía una consideración en el análisis a los aspectos relacionados al género de la población en estudio, más allá de si los estudios o intervenciones consideraban hombres o mujeres en sus diseños.

La Figura 7 muestra que de los estudios realizados en población masculina (15 estudios) en el 100\% de los casos no se realizó algún análisis de género para explicar los resultados. Contrariamente, en el caso de los estudios realizados en población femenina (8 estudios) en un 63\% de los casos (5 estudios) sí se realizó un análisis de género para explicar los resultados o en la discusión, aun cuando este análisis en la mayoría de los casos era breve. En el caso de los estudios donde la población era mixta (9 estudios), solo en un $22 \%$ de los casos (2 estudios) se realizó un análisis de género.
Figura 7.

Consideración de análisis de género en las publicaciones según tipo de población bajo estudio.

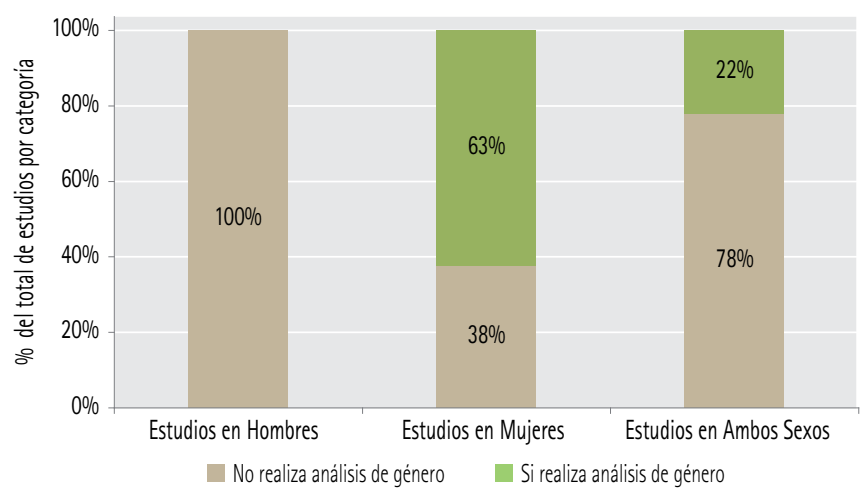

Tabla 1.

Estudios realizados en poblaciones de trabajo masculinizadas según sector de actividad.

\begin{tabular}{|c|c|c|}
\hline Sector & Autor(es) & Sujeto de estudio \\
\hline \multirow[t]{4}{*}{ Forestal } & $\begin{array}{l}\text { Apud E. } \\
\text { et al } 2002\end{array}$ & $\begin{array}{l}\text { Exigencias fisicas elevadas en el trabajo de bomberos } \\
\text { forestales. }\end{array}$ \\
\hline & $\begin{array}{l}\text { Apud E } \\
\text { et al. } 2003\end{array}$ & $\begin{array}{l}\text { Diseño de sistemas de organización en incendios } \\
\text { forestales y su relación con la carga de trabajo fisico. }\end{array}$ \\
\hline & $\begin{array}{l}\text { Chamblás L. } \\
\text { et al } 2005\end{array}$ & $\begin{array}{l}\text { Riesgos de accidentes y exigencias en el uso de serruchos } \\
\text { trozadores y finger-joint en una Empresa Maderera. }\end{array}$ \\
\hline & $\begin{array}{l}\text { Apud E y } \\
\text { Meyer F. } 2011\end{array}$ & $\begin{array}{l}\text { Analiza en profundidad los factores que influencian la } \\
\text { elevada carga de trabajo fisico en bomberos forestales. }\end{array}$ \\
\hline \multirow[t]{6}{*}{ Industrial } & $\begin{array}{l}\text { Espejo C. } \\
\text { et al } 2006\end{array}$ & $\begin{array}{l}\text { Evaluación de un dispositivo técnico-mecánico para } \\
\text { manipulación manual de cargas, para disminuir las } \\
\text { exigencias músculo-esqueléticas de los operadores. }\end{array}$ \\
\hline & $\begin{array}{l}\text { Quiceno L. } \\
\text { et al } 2006\end{array}$ & $\begin{array}{l}\text { Implementación de un programa de control y prevención } \\
\text { de la incidencia del síndrome de dolor lumbar asociado al } \\
\text { manejo manual de cargas. }\end{array}$ \\
\hline & $\begin{array}{l}\text { Córdova V. } \\
\text { et al } 2009\end{array}$ & $\begin{array}{l}\text { Determinar la capacidad de manejo manual de carga en } \\
\text { trabajadores chilenos, para determinar pesos máximos } \\
\text { aceptables para tareas de levantamiento, a través de } \\
\text { aproximación psicofísica. }\end{array}$ \\
\hline & $\begin{array}{l}\text { Córdova V. } \\
\text { et al } 2010\end{array}$ & $\begin{array}{l}\text { Percepción del peso de una carga en población laboral } \\
\text { masculina a través de una aproximación psicofisica. }\end{array}$ \\
\hline & $\begin{array}{l}\text { Cerda E. } \\
\text { et al } 2011\end{array}$ & $\begin{array}{l}\text { Medidas antropométricas de la mano y fuerzas de prensión } \\
\text { en trabajadores para obtener una muestra piloto que } \\
\text { permita orientar el diseño y la adquisición } \\
\text { de herramientas manuales. }\end{array}$ \\
\hline & $\begin{array}{l}\text { Pinto R. } \\
\text { et al } 2012\end{array}$ & $\begin{array}{l}\text { Determinación de ecuaciones de empuje y arrastre manual } \\
\text { de cargas a través de ensayos dinamométricos. }\end{array}$ \\
\hline \multirow[t]{5}{*}{ Minería } & $\begin{array}{l}\text { Solari G } \\
\text { et al } 2010\end{array}$ & $\begin{array}{l}\text { Estudio de la condición física en mineros y su vinculación } \\
\text { con la prevalencia de dolor lumbar. }\end{array}$ \\
\hline & Sandoval P. & Percepción de los trabajadores en sistemas de turno de 8 y \\
\hline & 2011 & 12 horas en faenas mineras. \\
\hline & $\begin{array}{l}\text { Oñate } \mathrm{E} . \\
\text { et al } 2012\end{array}$ & $\begin{array}{l}\text { Antropometria para el diseño de ropa de trabajo y } \\
\text { herramientas en mineria. }\end{array}$ \\
\hline & $\begin{array}{l}\text { Oñate E. y } \\
\text { Meyer F. } 2012\end{array}$ & $\begin{array}{l}\text { Exigencias fisicas, ambientales y carga mental de trabajo } \\
\text { en operador de planta de molibdeno. }\end{array}$ \\
\hline Servicios & $\begin{array}{l}\text { Freire J. } \\
\text { et al } 2012\end{array}$ & Carga de trabajo fisico en bomberos voluntarios de ciudad. \\
\hline
\end{tabular}

En la Tabla 1 se observan los estudios en sectores masculinizados; se puede constatar que en el sector forestal, el foco principal de las investigaciones ha sido mostrar las exigencias de trabajo físico, carga de trabajo, diseño y organización del combate de incendios forestales $^{10-12}$, así como el uso de herramientas para talar árboles. ${ }^{13}$ En el sector industrial se ha concentrado la mayor parte de los estudios, los cuales han tenido especial interés en los aspectos relacionados al manejo manual de cargas, en lo referente a métodos de 
evaluación, dispositivos técnicos de ayuda ${ }^{14-18}$ y la antropometría de mano para el diseño de herramientas. ${ }^{19}$ Otro sector importante es la minería, donde se han descrito las exigencias de trabajo en sistemas de turno ${ }^{20}$, los trastornos músculo-esqueléticos de espalda ${ }^{21}$, la carga mental y la antropometría para el diseño de ropa de trabajo. ${ }^{22,23}$

En el caso de los estudios realizados en poblaciones de trabajadoras, como se observa en la Tabla 2, es en los sectores de servicios donde se han realizado más investigaciones, en Salud ${ }^{24-26}$, en temas relacionados a la manipulación manual de pacientes y cargas, en algunos casos comparando las exposiciones con el sector industrial. En la industria del salmón, respecto de los efectos en la productividad, calidad y los trastornos músculoesqueléticos en trabajadoras ${ }^{27}$ producto del trabajo prolongado de pie, los esfuerzos repetitivos de elevada frecuencia de las extremidades superiores, en labores como el despinado y fileteado de salmón. Cabe destacar que solo un estudio aborda el tema del embarazo: en una muestra de 30 mujeres embarazadas laboralmente activas, estas presentaron en un 56\% algún tipo de percepción de molestias músculo-esqueléticas, las cuales se asociaban a una elevada carga mental y temporal en las actividades de trabajo que realizaban. ${ }^{28}$

Tabla 2.

Estudios realizados en poblaciones de trabajo feminizadas según sector de actividad.

\begin{tabular}{|c|c|c|}
\hline Sector & Autor(es) & Sujeto de estudio \\
\hline Salud & $\begin{array}{l}\text { Pinto, R. } \\
\text { et al } 2008\end{array}$ & $\begin{array}{l}\text { Comparación de las exigencias biomecánicas entre } \\
\text { diferentes métodos de transferencia en el manejo manual } \\
\text { de pacientes a través de electromiografia. }\end{array}$ \\
\hline $\begin{array}{l}\text { Salud, } \\
\text { Retail, } \\
\text { Agricultura } \\
\text { y Servicios }\end{array}$ & $\begin{array}{l}\text { Gutiérrez M. } \\
\text { et al } 2010\end{array}$ & $\begin{array}{l}\text { Analiza la prevalencia de los trastornos músculo-esqueléticos } \\
\text { de columna lumbar en trabajadoras de distintos rubros y los } \\
\text { limites biomecánicos en el manejo de carga y de pacientes. }\end{array}$ \\
\hline $\begin{array}{l}\text { Industrial; } \\
\text { Salud }\end{array}$ & $\begin{array}{l}\text { Córdova V. } \\
\text { et al } 2011\end{array}$ & $\begin{array}{l}\text { Comparación de la percepción del peso de una carga en } \\
\text { población laboral femenina industrial y salud. }\end{array}$ \\
\hline Servicios & $\begin{array}{l}\text { Gutiérrez M. } \\
\text { et al } 2008 \\
\text { Gutiérrez M. } \\
\text { et al } 2009\end{array}$ & $\begin{array}{l}\text { Capacidad de manejo de carga de trabajadoras y regulación } \\
\text { de peso máximo de carga humana. Ley № } 20.001 \text {. } \\
\text { Capacidad de manejo de carga de trabajadoras. }\end{array}$ \\
\hline Retail & $\begin{array}{l}\text { León M. } \\
2012\end{array}$ & $\begin{array}{l}\text { Muestra las exigencias músculo-esqueléticas y de salud } \\
\text { mental en cajeras del retail. }\end{array}$ \\
\hline $\begin{array}{l}\text { Salmoni- } \\
\text { cultura }\end{array}$ & $\begin{array}{l}\text { Ilardi, J. } \\
2012\end{array}$ & $\begin{array}{l}\text { Analiza la relación entre la productividad, calidad y los } \\
\text { trastornos músculo-esqueléticos en trabajadoras de la } \\
\text { industria del salmón. }\end{array}$ \\
\hline $\begin{array}{l}\text { Todos los } \\
\text { sectores }\end{array}$ & $\begin{array}{l}\text { Rodriguez C. } \\
\text { et al } 2011\end{array}$ & $\begin{array}{l}\text { Estima la carga global de trabajo desde el punto de vista de } \\
\text { la carga mental y la percepción de molestias músculo- } \\
\text { esqueléticas en mujeres embarazadas activas laboralmente. }\end{array}$ \\
\hline
\end{tabular}

Por otra parte, la Tabla 3 muestra los estudios realizados en poblaciones de trabajadores mixtas. Predominan los estudios en el sector salud, donde se abordan los problemas relacionados al trabajo en sistemas de turnos y la prevalencia de trastornos músculo-esqueléticos en trabadores/as que realizan manejo manual de pacientes y cargas ${ }^{29,30} \mathrm{y}$, más recientemente, las exigencias músculo-esqueléticas relacionadas al trabajo de los odontólogos. $^{31}$

Una serie de otras actividades han sido estudiadas en diferentes sectores como la salmonicultura ${ }^{32}$, la pesca, específicamente el procesamiento de crustáceos $^{33}$ y la agricultura ${ }^{34}$, donde el factor común es el estudio de la exposición a factores de riesgo por trabajo repetitivo, estando los hombres presente al inicio y al final de las líneas de producción, donde se requiere el uso de la fuerza $\mathrm{y}$ las mujeres en el medio de la línea donde se requiere rapidez y minuciosidad para no dañar la materia prima de exportación. Se encontró una publicación que aborda el tema de los riesgos en el trabajo en oficinas en relación a la utilización de computadores y los síntomas de trastornos músculo-esqueléticos ${ }^{35}$, además de una publicación en el sector de minería que incluye hombres y mujeres, centrándose en los aspectos de la carga de trabajo físico, las exigencias de carga mental y aspectos organizacionales, la antropometría y los factores de riesgo músculo-esqueléticos; no obstante, la población predominante es masculina.

Tabla 3.

Estudios realizados en poblaciones de trabajo mixtas según sector de actividad.

\begin{tabular}{|c|c|c|}
\hline Sector & Autor(es) & Sujeto de estudio \\
\hline \multirow[t]{3}{*}{ Salud } & $\begin{array}{l}\text { Córdova V. } \\
\text { et al } 2006\end{array}$ & $\begin{array}{l}\text { Estudia el impacto sobre la salud, vida social y rendimiento } \\
\text { individual del cuarto turno, determinando si existen } \\
\text { diferencias entre el personal que se desempeña en el área } \\
\text { pública y privada. }\end{array}$ \\
\hline & $\begin{array}{l}\text { Gutiérrez M. } \\
\text { et al } 2010\end{array}$ & $\begin{array}{l}\text { Prevalencia de los trastornos músculo-esqueléticos en } \\
\text { trabadores/as de centros hospitalarios que realizan } \\
\text { manipulación manual de pacientes y caracterización de } \\
\text { los factores de riesgo. }\end{array}$ \\
\hline & $\begin{array}{l}\text { Vallejos } \mathrm{H} \text {. } \\
\text { et al } 2011\end{array}$ & $\begin{array}{l}\text { Estudia el dolor músculo- esquelético relacionado al trabajo } \\
\text { de los odontólogos. }\end{array}$ \\
\hline Todos & $\begin{array}{l}\text { Muñoz C. } \\
\text { et al } 2012\end{array}$ & $\begin{array}{l}\text { Analiza los factores de riesgo disergonómicos y su relación } \\
\text { con el dolor lumbar a través del análisis de las bases de } \\
\text { datos de la encuesta nacional ENETS 2009-2010. }\end{array}$ \\
\hline $\begin{array}{l}\text { Salmoni- } \\
\text { cultura }\end{array}$ & $\begin{array}{l}\text { Apud E. } \\
\text { et al } 2003\end{array}$ & $\begin{array}{l}\text { Estudia las condiciones de trabajo en plantas salmoneras, los } \\
\text { factores de riesgos músculo-esqueléticos, la organización de } \\
\text { trabajo, las herramientas de trabajo y las características } \\
\text { antropométricas de los trabajadores/as. }\end{array}$ \\
\hline Pesca & $\begin{array}{l}\text { Sáez V. } \\
\text { et al } 2004\end{array}$ & $\begin{array}{l}\text { Muestra los factores de riesgos músculo-esqueléticos por } \\
\text { trabajo repetitivo y postura de pie en trabajadores/as de una } \\
\text { planta procesadora de crustáceos. }\end{array}$ \\
\hline Agric & $\begin{array}{l}\text { Castellucci, l. } \\
\text { et al } 2008\end{array}$ & $\begin{array}{l}\text { Analiza los factores de riesgos músculo-esquelético en } \\
\text { trabajadores/as que realizan actividades con trabajo } \\
\text { repetitivo, manipulación manual de cargas en un packing } \\
\text { de una empresa productora de champiñones. }\end{array}$ \\
\hline Servicios & $\begin{array}{l}\text { Muñoz C. } \\
\text { et al } 2012\end{array}$ & $\begin{array}{l}\text { Muestra los factores de riesgo presentes en el trabajo en } \\
\text { oficinas con la utilización de computadores y su relación } \\
\text { con los sintomas músculo-esqueléticos. }\end{array}$ \\
\hline Minería & $\begin{array}{l}\text { Apud E. } \\
2012\end{array}$ & $\begin{array}{l}\text { Análisis de varios estudios de caso de } 700 \text { centros de trabajo } \\
\text { en minería que incluyen hombres y mujeres respecto de la } \\
\text { carga de trabajo físico, exigencias mentales y } \\
\text { organizacionales, antropometria y factores de riesgo } \\
\text { músculo-esqueléticos }\end{array}$ \\
\hline
\end{tabular}

Otro de los aspectos importantes a considerar en ergonomía, sobre todo para el diseño y rediseño de puestos de trabajo, son las bases de datos antropométricos de la población de trabajadores/as. En la Figura 8 podemos observar una de las pocas bases de datos antropométricos publicadas oficialmente con datos de hombres y mujeres, correspondiente a 3.765 trabajadores/as chilenos/as. ${ }^{32}$ Las diferencias observadas entre los percentiles $5 \mathrm{y}$ 95 de los hombres representan $22 \mathrm{~cm}$ en estatura y $3,5 \mathrm{~cm}$ en el largo de la mano. Mientras que las diferencias entre los valores promedio de los hombres y de las mujeres es solo de 13,9 cm para la estatura y de $1,2 \mathrm{~cm}$ para el largo de la mano. Lo anterior muestra que las diferencias entre los percentiles extremos de los hombres son mayores a las diferencias existentes entre los valores comparados para el percentil 5,95 y promedio entre hombres y mujeres.

Profundizando en los hallazgos de los estudios de manipulación manual de cargas, el estudio realizado por Córdova et al 2011 
Figura 8.

Caracteristicas antropométricas de 2030 hombres 1735 mujeres chilenos/as entre 17 y 60 años de edad. Apud E. y cols. 2003.

\begin{tabular}{|l|c|c|c|}
\hline \multicolumn{3}{|c|}{ Estatura (cm) } \\
\hline & $\mathbf{p 5}$ & Promedio & $\mathbf{p 9 5}$ \\
\hline Mujeres & 144,8 & 154,9 & 165 \\
& \multicolumn{1}{|c|}{$20,2 \mathrm{~cm}$} & $13,9 \mathrm{~cm}$ \\
\hline Hombres & 157,8 & 168,8 & 179,8 \\
& \multicolumn{2}{|c|}{$22 \mathrm{~cm}$} & \\
\hline
\end{tabular}

\begin{tabular}{|c|c|c|c|}
\hline \multicolumn{4}{|c|}{ Largo de la mano $(\mathrm{cm})$} \\
\hline & p5 & Promedio & p95 \\
\hline \multirow[t]{2}{*}{ Mujeres } & 15,3 & 16,7 & 18,1 \\
\hline & & $2,8 \mathrm{~cm}$ & $1,2 \mathrm{~cm}$ \\
\hline \multirow[t]{2}{*}{ Hombres } & 16,4 & $18,1]$ & 19,9 \\
\hline & & $3,5 \mathrm{~cm}$ & \\
\hline
\end{tabular}

(Tabla 4) muestra que las mujeres del sector salud chileno tienen una percepción de exigencia de la carga manipulada más baja que aquellas mujeres del sector industrial, ya que estas consideran como muy pesada una carga de $51 \mathrm{Kg}$ y máxima cuando es mayor a $100 \mathrm{Kg}$, en comparación a las mujeres del sector industrial, que consideran como muy pesada una carga de $16 \mathrm{Kg}$ y máxima cuando es mayor a $42 \mathrm{Kg}$. Esto refleja el hecho de que para el mismo sexo la percepción del esfuerzo puede variar notablemente, lo que denota las diferencias que se producen al trabajar en un sector masculinizado (industrial) a otro feminizado (salud).

\section{Tabla 4}

Cantidad de carga para las categorias lingüisticas especificadas que le corresponde un nivel de pertenencia máximo, para mujeres del sector salud e industrial chileno (Córdova et al 2011).

$\begin{array}{lcc}\text { Categoría lingüistica } & \text { Sector Industrial }(\mathrm{Kg}) & \text { Sector Salud }(\mathrm{Kg}) \\ \text { Moderada } & 5 & 26 \\ \text { Pesada } & 11 & 41 \\ \text { Muy Pesada } & 16 & 51 \\ \text { Máxima } & 42 & 1100\end{array}$

Los mismos autores ${ }^{16}$, muestran que 27 hombres de una empresa del sector industrial chileno (Tabla 5), tienen una percepción de exigencia de la carga manipulada que es menor, comparativamente a las mujeres del mismo sector industrial siendo considerada por los hombres una carga de $81,5 \mathrm{Kg}$ como muy pesada, mientras que para las mujeres sobre los $42 \mathrm{Kg}$.

Tabla 5.

Cantidad de carga para las categorias lingüísticas especificadas de percepción del esfuerzo en población industrial masculina (Córdova et al 2010).

\begin{tabular}{|lcc|}
\hline Nivel & Categoría lingüística & Media $(\mathrm{Kg})$ \\
\hline 1 & Despreciable & 0,8 \\
\hline 2 & Muy, muy liviana & 2,6 \\
3 & Muy liviana & 5,2 \\
4 & Liviana & 8,5 \\
\hline 5 & Moderada & 14,9 \\
\hline 6 & Algo pesada & 23,3 \\
\hline 7 & Pesada & 33,9 \\
\hline 8 & Muy Pesada & 48,2 \\
\hline 9 & Muy, muy Pesada & 64,8 \\
\hline 10 & Máxima & 81,5 \\
\hline
\end{tabular}

Diferente es el caso de las mujeres del sector salud que perciben una carga como máxima cuando esta supera los $100 \mathrm{Kg}$, sobrepasando el valor referido por los hombres del sector industrial.

\section{DISCUSIÓN}

Esta revisión ha mostrado que más allá de lo que reflejan las escasas estadísticas oficiales en términos de accidentes y enfermedades, es conocido que en términos generales los hombres presentan mayores tasas de accidentes -y, en especial, de accidentes fatales- que las mujeres ${ }^{36}$; además, los hombres en los países en desarrollo presentan mayor exposición que las mujeres a ruido, vibraciones, ambientes extremos por temperatura, altura geográfica, trabajo en condiciones hiperbáricas en el mar, exposición a químicos tóxicos y manipulación de cargas pesadas, lo que se explica por la división sexual del trabajo. Es por esto que muchas sociedades aceptan la idea de que el hombre puede realizar trabajos más peligrosos.

Este estereotipo de género ha afectado, por ejemplo, las investigaciones en materia de salud reproductiva en hombres, dado que este tema ha sido visto como un dominio casi exclusivo de las mujeres, despreciando la importancia de la exposición ocupacional de los hombres. ${ }^{37-38}$ Ahora, uno de los efectos más nefastos que tienen estos estereotipos de género ha sido la invisibilidad de los riesgos en el trabajo de las mujeres tanto para la investigación como para las políticas públicas y la atribución casi sistemática de sus enfermedades a cuestiones ligadas al sexo, sin profundizar previamente en cuál es la actividad de trabajo y los diversos riesgos que pueden estar presentes, como hemos constatado en esta revisión a pesar de los pocos estudios en sectores feminizados.

Uno de los factores relevantes en el contexto de la investigación y el desarrollo de políticas en salud del trabajo es el hecho de que muchas veces se parte del supuesto equivocado de que el trabajo que realizan las mujeres es seguro. Este aspecto ha sido analizado por la investigadora Karen Messing ${ }^{39}$, para lo cual ella propone como explicación a este problema el círculo vicioso de la investigación sobre la salud de las mujeres en los lugares de trabajo (Figura 9). El supuesto de que el trabajo que realizan las mujeres no es riesgoso hace que no se investigue el trabajo que ellas realizan, no se demuestran los riesgos, se produce una falta de incentivo a la investigación, una ausencia de recursos para investigación y, por lo tanto, un desconocimiento de los riesgos.

\section{Figura 9.}

El circulo vicioso de la investigación sobre la salud de las mujeres en los lugares de trabajo (Messing 1998).

\section{Suposición: El trabajo de las mujeres es seguro}
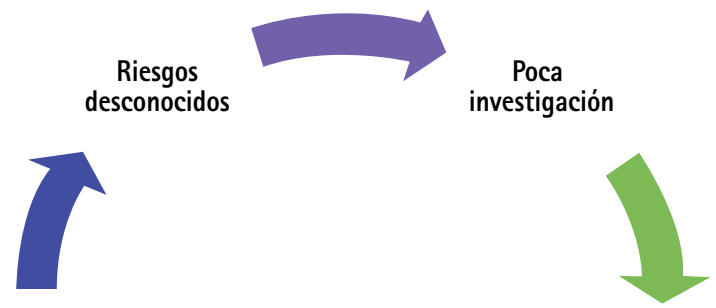

\section{Ausencia de recursos para la investigación}

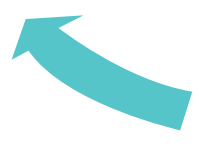

Falta de incentivo a la investigación

\section{.}


El impacto de estas suposiciones las encontramos en Chile, por ejemplo, en la tabla de cotización adicional genérica (diferenciada) del DS $\mathrm{N}^{\circ} 110 / 68$ del MINTRAB, donde para los sectores servicios y comercio es de $0 \%$, así como en lavanderías y tintorerías es de 0,85\%. A diferencia de lo que ocurre por ejemplo en minas y canteras donde la cotización es de 3,4\% (la máxima según el decreto). Esto evidentemente representa un incentivo negativo a la prevención de riesgos en estos sectores de actividad, donde trabajan principalmente mujeres, considerando que al partir de un riesgo presunto inexistente no se generan mecanismos por parte de las empresas, las mutualidades y los organismos fiscalizadores, que les incentiven a desarrollar políticas de prevención de riesgos a la salud de trabajadoras y trabajadores en estas actividades. Considerando además que esta cotización genérica influye en el criterio de exigencia para el tiempo de permanencia del experto en prevención de riesgos (en días a la semana) en la empresa, según lo establecido en el art 11 del DS N 40/69 del MINTRAB. Lo anterior se suma a la falta de reconocimiento de enfermedades profesionales, sobre todo las de origen músculo-esquelético o las de salud mental, que en Chile es evidenciado por la falta de información oficial al respecto en las bases de datos de acceso público.

Por otra parte, es evidente que la conciliación de trabajo y familia afecta mayoritariamente a las mujeres trabajadoras, evidenciando la doble carga de trabajo que ellas tienen ${ }^{9}$, producto de la construcción social que ha modelado las responsabilidades de la crianza de los hijos y las responsabilidades domésticas más allá del rol reproductivo ligado al sexo. De hecho, la tasa de fecundidad de las mujeres chilenas laboralmente activas ha caído hasta 1,2 hijos. ${ }^{40}$ Para ellas es un desafío particular el equilibrio entre la salud en el trabajo y la igualdad profesional ${ }^{41}$, pero ello no significa que sus problemas de salud no están ligados al trabajo. Por esto es necesario estudiar estos trabajos para conocer los riesgos y ayudar a mejorar las condiciones de trabajo de todos los trabajadores/as, considerando que el empleador tiene la responsabilidad de garantizar que las condiciones de trabajo y empleo permitan una buena conciliación. Su parte concierne a los horarios de trabajo, la posibilidad de acceder al teléfono cuando es necesario, las pausas y la posibilidad de volver a casa después del trabajo en un estado físico y mental que permita a la persona estar presente con su familia y sus amigos. ${ }^{42}$

Otro aspecto importante que reflejó la revisión de las bases de datos gubernamentales son los resultados de la calificación de trabajos pesados por la Ley 19.404, instancia que, por cierto, ha permitido el desarrollo de la ergonomía en Chile. Se debe tener en cuenta que esta ha sido una de las instancias históricas de utilización de la ergonomía como un medio de justicia social, dado que los trabajadores/as acceden a través de esta calificación al sistema de jubilación anticipada. No obstante, han sido las mujeres quienes menos han accedido a estos beneficios.

En relación a las publicaciones realizadas por ergónomos chilenos y la pregunta sobre cuál ha sido el rol de la ergonomía en el proceso de comprender las diferencias de sexo y género, en términos de salud y seguridad en el trabajo: en general, son pocos los estudios que han considerado aspectos de sexo y género en sus publicaciones. Es importante considerar que la información parcial puede aumentar las inequidades de género en cuanto a las posibilidades de inserción para las mujeres en medios de trabajo tradicionalmente masculinos o bien en las condiciones de trabajo y acceso a la prevención de enfermedades y accidentes, el uso de herramientas manuales o la ropa de trabajo, entre otros. Estos aspectos son relevantes en el sentido de que existen políticas gubernamentales que buscan aumentar la inserción laboral femenina en medios de trabajo no tradicionales para las mujeres, como la minería y la construcción Ej: Programa Mujer Minera, SERNAM 2012, para lo cual los ergónomos serán llamados a identificar estas brechas y ayudar desde el punto de vista técnico a adaptar el trabajo a una población de utilizadores/as que comenzará a ser más variable desde múltiples puntos de vista.

Tener en cuenta las características personales de cada trabajador/a tiene sus raíces en la práctica de la ergonomía, considerando: las características antropométricas, las variaciones morfológicas, los efectos de la edad y el envejecimiento, la vuelta al trabajo de las personas con discapacidades funcionales $\mathrm{y}$, evidentemente, el sexo y el género. A menudo se tienen en cuenta las diferencias ligadas al sexo, pero muy poco al género ${ }^{43}$, como fue evidenciado en esta revisión donde por cierto los análisis de género se asocian principalmente a los estudios en poblaciones feminizadas, contrariamente a lo que ocurre en las poblaciones de trabajo masculinizadas. La falta de consideración de los aspectos ligados al sexo y al género de las trabajadoras/es en las investigaciones o intervenciones que realizan los ergónomos puede explicarse por el hecho de que no es fácil abordar las cuestiones de género en una intervención ergonómica, que busca responder a la petición de una empresa cuyo interés en la prevención de riesgos de salud y seguridad en el trabajo, a menudo, es nuevo y frágil. Por lo tanto, el interés en las cuestiones de género por lo general es nulo. ${ }^{43}$ No obstante lo anterior, las publicaciones analizadas en esta revisión han permitido comprender mejor cuáles son las principales dificultades que hombres y mujeres deben afrontar en sus lugares de trabajo.

Es necesario considerar que una intervención en ergonomía se construye en función del contexto social de la empresa, por lo tanto, es primordial evaluar este contexto (nivel de sensibilización) y elaborar estrategias de intervención en función de aquello que los actores de la empresa están preparados a entender. Pero también se construye en función del cambio de representación que los actores concernientes en el medio de trabajo tienen sobre la actividad que realizan las trabajadoras/es, lo cual debe representar un objetivo de la intervención buscado por el ergónomo, para adaptar el trabajo a las personas considerando su variabilidad. La sensibilización en la prevención de los riesgos representa ya un tema importante, por lo que la sensibilización a los aspectos de sexo y de género puede parecer difícil en ciertos contextos. ${ }^{43-45}$

Dado que los hombres y las mujeres están distribuidos de forma diferenciada en los lugares de trabajo a causa de la división sexual del trabajo y los estereotipos de género, como fue constatado en esta revisión, ellos presentan exposiciones diferentes a los riesgos aun cuando trabajen en la misma empresa o faena. Es por esto que algunos ergónomos comienzan a preguntarse cuál es la importancia que se debe atribuir al sexo en los estudios ergonómicos. ${ }^{39,41,42,46-48}$ Los puestos de trabajo y las actividades varian según el sexo, lo que implica la pertinencia de considerarlo para comprender mejor los modos operatorios, por lo cual la consideración del sexo de los trabajadores/as no se reduce solamente a una categoría, sino que esto proporciona una manera de enriquecer el análisis ergonómico. ${ }^{46}$

Si no hay un análisis fino de todos los componentes de la actividad de trabajo, los científicos que observan que las trabajadoras y trabajadores no tienen las mismas enfermedades concluirán que se esto se debe a los aspectos hormonales, debilidades asociadas a 
un sexo o una tendencia a quejarse. Para el ergónomo, será siempre pertinente tener en cuenta el sexo de los trabajadores/as en diferentes instancias de la intervención. ${ }^{46}$ Los estudios ergonómicos pueden aportar una evidencia clara acerca de la necesidad de pasar de los análisis macro (epidemiológicos, sociológicos) a un nivel micro ${ }^{47}$, donde las asociaciones entre los riesgos y los determinantes se hacen visibles más allá de los aspectos ligados al sexo de los trabajadores/as y los estereotipos de género.

Dado lo anteriormente descrito, es recomendable realizar estudios de mujeres en sectores masculinizados (construcción, transporte, minería, buceo, etc.) y de hombres en sectores feminizados (servicios sanitarios, educacionales, etc.) para documentar dificultades que pueden representar barreras de inserción ${ }^{48}$, además considerar que en todo contexto, feminizado, masculinizado o mixto, las particularidades de sexo y género están siempre presentes y es importante comprender la variabilidad que esto genera para poder realizar transformaciones sustentables. Comprender la variabilidad es necesario respecto de la identificación de la exposición a diferentes factores de riesgo, pero también en cuanto a las relaciones funcionales y jerárquicas que se desarrollan en las empresa, reflexionando sobre hasta qué punto las representaciones de los trabajadores/as y empleadores/as corresponden a aspectos de sexo o bien si estas corresponden a estereotipos de género. Otro aspecto importante a considerar en investigaciones e intervenciones futuras es la conciliación de trabajo y familia, respecto de los efectos del trabajo de manera diferenciada para hombres y mujeres.

Es recomendable en toda intervención considerar que -al momento de analizar la demanda de intervención-, los hombres y mujeres pueden tener intereses diferentes, aun cuando se trate del mismo trabajo, ellos/as pueden tener problemas de salud diferentes en función de las operaciones o los modos operatorios característicos a cada uno, dada sus diferencias biológicas y a la división sexual del trabajo. ${ }^{46-47}$ Como las mujeres habitualmente están excluidas de los puestos de trabajo donde los peligros son impresionantes y dramáticos (como en el caso de los bomberos), será fácil subestimar sus dificultades y riesgos (como en el caso de la manipulación manual de cargas en mujeres del sector salud). Por lo tanto, en las entrevistas con los supervisores es muy importante preguntar sobre las dificultades para los dos sexos y realizar entrevistas separadas a hombres y mujeres. En las observaciones, deben identificarse los modos operatorios específicos para cada sexo a través de los cuales pueden encontrarse los determinantes. Será importante identificar las habilidades desconocidas que son implementadas por las trabajadoras/es durante las operaciones aparentemente triviales y que no son fácilmente visibles para quienes se aproximan a analizar el trabajo; por ejemplo: en la industria agroalimentaria no es del todo valorado el cómo sacar las espinas de un salmón, cómo seleccionar la uva de mesa, cómo atender un cliente conflictivo. Esto muchas veces es referido en el discurso de la empresa como el valor agregado de la producción de bienes o de servicios, pero a nivel organizacional no es reco- nocido, por lo tanto, las demandas físicas, mentales y emocionales, aparecen menos visibles.

Por otra parte, en el análisis de los resultados y en la búsqueda de soluciones será pertinente confrontar ciertos estereotipos de género que pueden obstaculizar la puesta en marcha de soluciones, sobre todo en medios de trabajo masculinizados o feminizados; en este caso es necesario combatir estos estereotipos, para lo cual puede ser pertinente implicar ergónomos de ambos sexos en la presentación de los resultados. ${ }^{46-47}$

Si la intervención implica realizar formación, puede ser recomendable ver con mayor atención aquello que las trabajadoras pueden aprovechar, puesto que frecuentemente se encuentran excluidas de las instancias decisionales en materia de salud y seguridad en el trabajo, como jefaturas, sindicatos, comités de higiene y seguridad. ${ }^{46}$

Finalmente, es importante remarcar el hecho de que en Chile no existe una revista que recopile el trabajo que realizan los ergónomos, lo cual sin duda ha representado un límite en este trabajo.

\section{CONCLUSIONES}

El tomar en cuenta el sexo de las trabajadoras/es enriquece el análisis del trabajo y el análisis ergonómico a su vez enriquece la comprensión sobre la división sexual del trabajo y cómo esta genera diferentes exposiciones a los riesgos profesionales para las mujeres y los hombres.

En Chile, claramente existe una exposición laboral a los riesgos en el trabajo que, en el caso de las mujeres, parece ser más importante que aquella que se conoce y si bien los hombres también se encuentran expuestos, las diferentes situaciones sociales han hecho que al menos sean más considerados en las políticas públicas de prevención de seguridad y salud en el trabajo.

Los ergónomos habitualmente toman en cuenta las características de los trabajadores (antropométricas, fisiológicas) en los lugares donde intervienen y, por lo tanto, ayudan a disminuir la discriminación sistemática que sufren las mujeres o los hombres a causa de sus particularidades. Sin embargo, poco se reflexiona sobre las diferencias sociales que pueden afectar la salud laboral de hombres y mujeres de manera diferenciada. Se debe considerar que los ergónomos serán llamados a hacerse cargo de estos aspectos, sobre todo en los denominados riesgos emergentes como los músculo-esqueléticos y los psicosociales.

Es importante considerar que las condiciones de trabajo no tienen sexo, por lo tanto, la búsqueda de los determinantes que explican los riesgos y las transformaciones de los lugares de trabajo, sustentadas en la comprensión de la variabilidad de sexo y de género de las trabajadoras/es, puede conducir a los ergónomos a intervenciones que representaran mejoras en las condiciones de trabajo para mujeres y hombres. 


\section{El tema ha sido presentado en 2 conferencias bajo el nombre de:}

1. Equidad de género y ergonomía en Chile: ¿Cuál es el rol del ergónomo? XI Congreso Internacional de Prevención de Riesgos Laborales ORP2013, Santiago de Chile 4 de abril de 2013.

2. Género, trabajo femenino y ergonomía en Chile: ¿Cuál es el rol del ergónomo? 8vo Congreso Chileno de Ergonomía y 1ra Jornada de Ergonomía Minera de la Sociedad Chilena de Ergonomía (SOCHERGO), 31 de agosto al 01 de Septiembre 2012, Antofagasta, Chile.

\section{Financiamientos:}

Este trabajo se presenta en el marco del proyecto de colaboración internacional "Ergonomía para la salud y el desarrollo sustentable en Chile”, financiado por la Agencia Canadiense para el Desa- rrollo Internacional (ACDI), gestado por la Universidad de Quebec en Montreal (UQÀM) y el Instituto de Salud Pública de Chile.

\section{AGRADECIMIENTOS}

Nuestros agradecimientos a l'Université du Québec à Montréal (UQÀM) y a la Agence Canadienne de Développement International (ACDI) por haber gestado y financiado respectivamente este proyecto de cooperación internacional. Así como al Instituto de Salud Pública de Chile (ISPCH), el Centro de Estudios de la Mujer (CEM) y la Sociedad Chilena de Ergonomía (SOCHERGO) que colaboraron con este proyecto en Chile. Agradecemos especialmente a la Dra. Nicole Vézina, Dra. Karen Messing, Dra. Ana María Seifert, Dra. Céline Chatigny de CINBIOSE y a la Dra. Florence Chappert de la ANACT, quienes nos apoyaron en este proceso.

\section{REFERENCIAS}

1. Apud E, Meyer F. Ergonomics in Chile. En: Scott PA. Ergonomics in developing regions: Needs and applications.Cap.27. Boca Ratón: CRC Press; 2010.

2. Figueroa ME. La ergonomia en Cile: tres décadas de desarrollo [en línea]. Santiago:Sochergo; [2011] [citado mar 2014]. Dosponible en: http://www. sochergo.cl/portal/articulos/61/libro-30-anos-de-ergonomia-en-chile.html.

3. Habib R, Messing M. Gender, women's work and ergonomics. Ergonomics. 2012;55(2):129-132.

4. Ergonomics. February 2012;55(2).

5. Laboreal. 2012;8(1).

6. Díaz E, Mella P. Inequidades y brechas de género en el empleo; Análisis de los resultados de la Encuesta Nacional de Coyuntura Laboral ENCLA 2008, Sexta Versión. Santiago: Dirección del Trabajo; 2009.

7. Chile. MINSAL. Encuesta Nacional de Calidad de Vida Informe Institucional ENCAVI 2006. Santiago: MINSAL; 2007.

8. Chile. MINSAL. Primera Encuesta Nacional de Empleo, Trabajo, Salud y Calidad de vida de los Trabajadores y Trabajadoras en Chile; Informe Interinstitucional ENETS 2009-2010. Santiago: MINSAL; 2011.

9. Vallebuona C, Hoffmeister L. Encuesta de Calidad de Vida y Salud: Resultados en población trabajadora. I Congreso Chileno de Salud Pública. Santiago: MINSAL; 2007.

10. Apud $E$, Meyer $F$, Maureira F. Ergonomía en el Combate de Incendios Forestales. Proyecto Fondef D9911972. Santiago: Universidad de ConcepciónACHS; 2002.

11. Apud E, Meyer F. La ergonomía como apoyo al diseño de sistemas de organización en incendios forestales. Revista Ação Ergonômica 2003;1(4):21-33.

12. Apud $E$, Meyer F. Factors influencing the workload of forest fire-fighters in Chile. Work. 2011;38(3):203-209.

13. Chamblás LA, Vargas JP. Estudio Ergonómico en Serruchos Trozadores y Finger-Joint en una Empresa Maderera. Cienc Trab.2005;7(18):140-147.

14. Pinto R, Córdova V, Quinceno L. Ecuaciones de empuje y arrastre ACHS: Relación entre el peso de una carga y la fuerza humana necesaria para moverla. Cienc Trab. 2012; 14(45):228-232.

15. Córdova V, Pinto R, Llambias J, Chávez B. Capacidad de Manejo Manual de Carga de Trabajadores Chilenos: Pesos Máximos Aceptables para Tareas de Levantamiento. Cienc Trab. 2009;11(34):204-210.

16. Córdova V, Troncoso R, Pinto R. Percepción del Peso de una Carga en Población Laboral Masculina Chilena: Modelamiento Basado en la Teoria de Conjuntos Difusos. Cienc Trab.2010;12 (37):370-375.

17. Espejo C, Mallea H, Azagra H.. Evaluación de un Elevador Neumático para Carga de Cabezal en Área de Printer, en una Empresa Productora de Tapas Plásticas en Chile. Cienc Trab. 2006;8(19):21-25.
18. Quinceno L, Celedón A. Implementación de un Programa de Control y Prevención de Sindrome de Dolor Lumbar Asociado al Manejo Manual de Cargas en la Planta de Pintura a Base de Agua en la Empresa Sherwin Williams, Santiago. Cienc Trab. 2006;8(19):11-15.

19. Cerda E, Cubillos N, Medina O, Rodríguez C. Estudio Piloto de Medidas Antropométricas de la Mano y Fuerzas de Prensión, Aplicables al Diseño de Herramientas Manuales. Cienc Trab. 2011; 13(39):1-5.

20. Sandoval P. Percepción de trabajadores en sistemas de turno de 8 y 12 horas. Cienc. Trab. 2011;13(40):92-101.

21. Solari G, Solari B. Identificación de Variables Relacionadas con la Condición Física para el Control Ergonómico de Factores Humanos Vinculados al Dolor Lumbar. Cienc Trab. 2010;12(38):454-460.

22. Oñate $E$, Meyer F. Ergonomic study of an operator _s work of a molybdenum plant. Work. 2012;41(Suppl.1):5950-5955.

23. Oñate $E$, Meyer $F$, Espinoza J. On the road toward the development of clothing size standards and safety devices for Chilean workers. Work. 2012;41(Suppl 1):5400-5402.

24. Pinto R, Córdova V, Silvestre R. Estudio de Caso: Comparación biomecánica entre métodos de transferencia en el Manejo Manual de Pacientes. VI Congreso Internacional de Prevención de Riesgos Laborales ORP;14-16 may 2008, Coruña, España. Barcelona: UPC; 2008.

25. Gutiérrez M, Flores $C$, Monzó J. Prevalencia de Trastornos MúsculoEsqueléticos en Funcionarios de Centros Hospitalarios que Realizan Manejo de Pacientes y Caracterización de Potenciales Factores de Riesgo. Cienc Trab.2010;12(38): 447-453.

26. Córdova V, Troncoso R, Pinto R. Comparación de la Percepción del Peso de una Carga en Población Laboral Femenina: Sector Industrial Versus Sector Salud. Cienc Trab. 2011;13(39):17-23.

27. Ilardi J. Relationship between productivity, quality and musculoskeletal disorder risk among deboning workers in a Chilean salmon industry. Work. 2012;41:5334-5338.

28. Rodríguez C, Gutiérrez L, Cortés M, Cerda E. Estudio de la Carga Global de Trabajo y Percepción de Molestias Músculo-Esqueléticas en Embarazadas Atendidas en la Unidad de Maternidad del Hospital Clínico de la Universidad de Chile. Cienc Trab. 2011;13(39):53-56.

29. Córdova V, Hevia JC, Figueroa A.Trabajo en turnos en el sector de la salud chileno: una comparación entre el sector público y privado. Cienc Trab. 2006;8(21):147-150.

30. Gutiérrez M, Flores $C$, Monzó J. Prevalencia de Trastornos MúsculoEsqueléticos de Columna Lumbar en Trabajadoras y Limites Biomecánicos en el Manejo de Carga y Pacientes. Cienc Trab. 2010; 12(37):380-385.

31. Vallejos $H$, Rodriguez $S$, Quintana S. 2011. Dolor Músculo-Esquelético en Alumnos de Postgrado en Rehabilitación Oral de la Facultad de Odontología 
de la Universidad del Desarrollo-Concepción. Estudio Piloto. Cienc Trab. 2011;13 (41):158-161.

32. Apud E, Lagos S, Maureira F. Estudio Ergonómico en Plantas Salmoneras de la X Región. Santiago: Dirección del Trabajo de Chile; 2003. (Cuaderno de Investigación; 17).

33. Sáez V, Arriagada C, Marco K, Manriquez 0 . Prevalencia de lesiones músculo- esqueléticas y factores de riesgo en trabajadores de plantas procesadoras de crustáceos en Chile. Cienc Trab. 2004;6(13):100-110.

34. Castellucci I, Robert P, Viviani CA. Estudio Ergonómico en el área de Packing de una Productora de Champiñones. VI Congreso Internacional de Prevención de Riesgos Laborales ORP;14-16 may 2008, Coruña, España. Barcelona: UPC; 2008.

35. Muñoz C, Vanegas J. Asociación entre puesto de trabajo computacional y sintomas musculoesqueléticos en usuarios frecuentes Med Segur Trab. 2012;58(227):98-106.

36. Messing K, Ostlin P. Gender equality work and health: a review of the evidence. Geneva: World Health Organization-Department of Gender, Women and Health; 2006. $54 \mathrm{p}$.

37. Wang $Y$. Male reproductive health research needs and research agenda: Asian and Pacific Perspective. Int J Androl. 2000;23(S2):4-7.

38. Varga CA. The forgotten fifty per cent: a review of sexual and reproductive health research and programs focused on boys and young men in subSaharan Africa. Afr J Reprod Health. 2001;5(3):175-195.

39. Messing K. La santé des travailleuses. La science est-elle aveugle?. Montréal: Remue-Ménage; 1998.

40. Jeri T. Perfil de las Trabajadoras en Chile. Santiago: Departamento de Estudios y Capacitación-Servicio Nacional de la Mujer; 2008. (Documento de Trabajo; 108).

41. Messing K. Santé des femmes au travail et égalité professionnelle: des objectifs conciliables? Travailler. 2009;2(22):43-58.

42 Prévost J, Messing K. Stratégies de conciliation d'un horaire de travail variable avec des responsabilités familiales. Le travail humain. 2001;64(2):119-143.

43. Vézina N, Tougas G. L'intervention ergonomique et la construction sociale: où se situe la préocupation du sexe/genre? Colloque du CINBIOSE sur le Genre, Santé et Environement. Montreal : CINBIOSE; 2012.
44. St-Vincent $M$, Vézina $N$, Bellemare $M$, Denis $D$, Ledoux $E$, Imbeau $D$. L'intervention en ergonomie. Qúebec: Éditions MultiMondes; 2011.

45. Brunet $R$, Presselin J, Viel $M$, Sée $N$. Le risque et la parole: construire ensemble une prévention des risques du travail dans l'agriculture et l'industrie. Toulouse : Octarès; 2005.

46. Messing K. La pertinence de tenir compte du sexe des" opérateurs" dans les études ergonomiques: Bilan de recherches. Pistes.1999;1(1):1-12.

47. Messing K. Comprender el trabajo de las mujeres para transformarlo: estudio de varios casos sobre la igualdad y la salud y seguridad en el trabajo. En: Messing K. El trabajo de las mujeres: comprender para transformar. Madrid: Los Libros de la Catarata; 2002. pp. 129-266.

48. Habib RR, El-Zein KE, Hojeij S. Hard work at home:musculoskeletal pain among female homemakers. Ergonomics. 2011;55(2):201-211.

\section{Referencias complementarias:}

Apud E, Meyer F. Ergonomía para la industria minera. Santiago: CODELCOUniversidad de Concepción; 2009.

Apud E. Ergonomics in mining: the Chilean experience. Hum Factors. 2012;54 (6):901-907.

Freire J, Meyer F, Apud E. Physical workload during firefighting in Chilean volunteers. Work. 2012;41:432-436.

Gutiérrez M, Flores C, Chesta A, Jofré N, Brito K, Monzó J. Capacidad de manejo de carga de trabajadoras y regulación de peso máximo de carga humana. Ley No 20.001. Rev Prev Riesgos. 2008;79:24-28.

Gutiérrez M, Flores C, Monzó J. Capacidad de Manejo de Carga de Trabajadoras. Cienc Trab. 2009;11(34):197-203.

León M. Participatory ergonomics among female cashiers from a department store. Work. 2012;41:2499-2505.

Muñoz C, Vanegas J, Marchetti N. Factores de riesgo ergonómico y su relación con dolor musculoesquelético de columna vertebral: basado en la primera encuesta nacional de condiciones de empleo, equidad, trabajo, salud y calidad de vida de los trabajadores y trabajadoras en Chile (ENETS) 2009-2010. Med Segur Trab. 2012;58(228):194-204. 